\title{
Prospective Evaluation of Handheld Ultrasound in the Diagnosis of Blunt Abdominal Trauma
}

\author{
A Brooks, B Davies, J Connolly
}

\begin{abstract}
Objectives

To evaluate the Sonosite 180 handheld ultrasound in the diagnosis of haemoperitoneum in blunt abdominal trauma.

Methods

Trauma ultrasound using the Focused Assessment with Sonar for Trauma (FAST) technique was performed using the Sonosite 180 handheld ultrasound during the primary survey of adult patients triaged to the resuscitation room with multiple trauma or suspected abdominal injury. The ultrasound findings were compared against the investigation of choice of the attending surgeon/accident \& emergency physician - CT, DPL, laparotomy or clinical observation.
\end{abstract}

Results

50 patients who had sustained blunt abdominal trauma were evaluated using FAST. Satisfactory images were obtained in $96 \%$. There were 5 true positive scans confirmed by CT, DPL or laparotomy. There were no false negative or false investigations. The sensitivity and specificity of handheld FAST was $100 \%$.

Conclusions

Handheld ultrasound using the Sonosite 180 system can be successfully used by appropriately trained doctors as the primary investigation in the acute evaluation of blunt abdominal trauma.

Capt A Brooks FRCS RAMC (V)

Specialist Registrar in General Surgery

Dept of Surgery

Queens Medical

Centre, University

Hospital, Nottingham, $\mathrm{NG} 72 \mathrm{O} 1 \mathrm{H}$

Email

adam.brooks@ukgateway.net

\section{Dr Davies}

Mr Connolly

Dept of Surgery and Accident \& Emergency Queens Medical Centre, University Hospital, Nottingham, NG7 2UH

\section{Introduction}

The evaluation of abdominal injury is challenging even in the hospital resuscitation room where specialist facilities including computerised tomography (CT) are readily available. In austere environments clinical examination is the mainstay of diagnosis. It is, however, often no more reliable than guess work, especially when there is associated head trauma, spinal injury or distracting injuries (1).

Unrecognised abdominal injuries and haemorrhage are a major source of preventable death in the trauma patient (2). Rapid and reliable investigation for haemoperitoneum is an essential part of the evaluation of all injured patients.

An ultrasound based technique for the evaluation of abdominal trauma - Focused
Assessment with Sonar for Trauma $($ FAST $)(3,4)$, was developed as a rapid and simple method for the assessment of the peritoneal cavity for bleeding. FAST has been shown to be reliable and sensitive for the assessment of blunt abdominal trauma (5). The development of hand portable ultrasound(6) has made ultrasound technology available for deployment. The Sonosite 180 is a hand carried ultrasound system that weighs only $2.4 \mathrm{~kg}$ and incorporates a $12.7 \mathrm{~cm}$ TFT liquid crystal display. The system is powered by an internal rechargeable lithium ion battery that lasts approximately 4 hours from full charge or from mains supply.

\section{Aim}

To prospectively evaluate the Sonosite 180 hand held ultrasound in the diagnosis of haemoperitoneum in blunt abdominal trauma.

\section{Methods}

Ethical approval for the study was granted by the Queens Medical Centre Ethics Committee reference GS040102.

Adult patients with multiple trauma or suspected blunt abdominal injury triaged to the resuscitation room from the 1st June 2001 for a six-month period were eligible for the study. Ultrasound assessment was undertaken using the Sonosite 180 hand carried ultrasound system using a 4-2 megahertz $15 \mathrm{~mm}$ curved array by one of two doctors $(\mathrm{AB}, \mathrm{JC})$ trained in the performance of FAST. The standard four-view FAST technique (perisplenic, perihepatic, pericardial, pelvis) as described by Rozycki (3) was used. FAST was undertaken during the circulation phase of the primary survey or early in the secondary survey (7). Patients who had sustained penetrating injury and those in extremis, where ultrasound examination would have led to a delay in definitive treatment, were excluded from the study. The ultrasound images were stored on the machine for review. The trauma team leader was informed of the FAST findings on completion of the examination to prevent ethical dilemmas. Data was recorded on the scan time and mechanism of injury.

The ultrasound findings were compared against the investigation of choice of the attending surgeon/accident \& emergency physician - CT, diagnostic peritoneal lavage (DPL), laparoscopy, laparotomy or clinical 
observation. The patients were followed until discharge for adverse events or late presentation of intra-abdominal injury.

\section{Results}

50 patients were assessed using the handheld ultrasound system and the FAST technique during the study period. The median injury severity score of the patients was 13 (range 1-75). The mechanism of injury sustained is shown in Table 1 . In 5 patients the assessment was technically incomplete, as all four views were not obtained adequately. In 2 of these patients the pericardial view was not seen, however, adequate abdominal views were obtained. In a further 3 patients gross surgical emphysema prevented adequate imaging of one view. The FAST examinations took no longer than 4 minutes to complete in all patients.

Table.1 Mechanism of injury sustained in the study patients

\begin{tabular}{|lc|}
\hline Motor vehicle crash & 20 \\
\hline Motorbike crash & 13 \\
\hline Fall from a height & 7 \\
\hline Pedestrian vs. vehicle & 4 \\
\hline Assault & 3 \\
\hline Other & 3 \\
\hline TOTAL & $\mathbf{5 0}$ \\
\hline
\end{tabular}

There were 5 positive FAST examinations in the study. Three of the patients underwent laparotomy, two following abdominal CT scan, that confirmed intra-abdominal haemorrhage. One patient had peritoneal haemorrhage confirmed by DPL, but suffered a cardiac arrest from which resuscitation was unsuccessful. The fifth patient arrested in the emergency room following a positive FAST, this patient had a massive head injury and resuscitation was therefore abandoned. The intra-abdominal bleeding was confirmed at post mortem. There were no false positive examinations.

FAST was negative for haemoperitoneum in 42 patients (including two patients where the pericardial view was not obtained). These findings were confirmed by CT in $47 \%$ and by clinical observation in the remainder. Follow up to hospital discharge confirmed that none proceeded to abdominal related morbidity or mortality during their inpatient stay. There were no false negative FAST examinations. The 3 technically incomplete examinations were augmented by CT in 2 patients and DPL in one patient, all were negative.

The sensitivity and specificity of FAST using handheld ultrasound was $100 \%$.

\section{Discussion}

The value of FAST as a technique for abdominal trauma assessment has previously been demonstrated in a number of large studies $(5,8,9)$. The results of these three papers, which combined have assessed more than 4000 injured patients, demonstrate a sensitivity of $86 \%-88.2 \%$ and specificity of $90 \%-99 \%$ for the detection of intraabdominal injuries using FAST.

This prospective evaluation of hand held ultrasound has shown that the FAST technique can be successfully applied using the Sonosite 180 ultrasound system as the primary investigation in the evaluation of abdominal trauma in the acute setting. The specificity and sensitivity of $100 \%$ for the detection of haemoperitoneum exceeds previous work where larger, less mobile ultrasound systems have been used. The high sensitivity found in this work may be a reflection of the relatively low number of positive examinations (5 patients) in the study population.

The main technical limiting factor for the acquisition of satisfactory images was surgical emphysema from chest injury. No technical factors attributable to the hand held system (screen size, image brightness etc) were recorded during the course of the study. Resuscitation room lights were rarely dimmed as this would have caused an interruption to the flow of the resuscitation and dynamic image quality was felt to be consistently sufficient for diagnosis despite the background lighting. No limiting factors attributable to the ultrasound operator were determined.

Patients who had sustained penetrating trauma were excluded form this study. FAST has previously been shown to have a low negative predictive value $(60 \%)$ in penetrating trauma, however it has a positive predictive value of $90 \%(10)$. Therefore FAST is valuable as an initial screening investigation in these patients but, if negative, the technique must be augmented by another modality.

The study was undertaken in the accident \& emergency department of a large teaching hospital (Queens Medical Centre, Nottingham) where more than 200 resuscitations for major trauma are undertaken annually. $10 \%$ of the FAST scans performed during the six-month study were positive. This is consistent with work by Bain(11) which found that only $10 \%$ of severely injured patients that were assessed by General Surgeons required abdominal surgery. It is important that surgeons/ physicians performing FAST maintain their skills, which may not be possible in institutions where less trauma is seen. Military surgeons and emergency physicians trained in FAST may require short predeployment refresher training if they are not in a situation to keep their ultrasound skills current.

The two investigators in the study had undertaken different FAST training. $\mathrm{AB}$ successfully completed the American College of Surgeons ultrasound for surgeons and 
ultrasound in the acute setting courses six months before starting the study. JC had attended a four-hour FAST ultrasound course that was followed by 10-proctored scans, including patients with ascites to simulate haemoperitoneum. The study results are equivalent to the findings of Thomas (12) who described $81 \%$ sensitivity, $99 \%$ specificity and $98 \%$ accuracy for surgeon-performed FAST after only an 8hour trauma US course and demonstrates that a short training period is adequate for successful FAST.

Handheld FAST ultrasound is a useful tool in the evaluation of abdominal trauma. The Sonosite 180 would be a valuable asset on deployment and FAST should be integrated into military casualty algorithms.

\section{Acknowledgements}

The authors would like to acknowledge the support for this project provided by the Drummond Foundation.

\section{References}

1. Krantz B. Secondary Assessment, in Current Therapy of Trauma, Lewis FR. Trunkey DD, Editor. 1999, Mosby: St Louis.

2. Anderson ID, Woodford M, Conbal FT, Irving $M$. Retrospective Study of 1000 trauma deaths from injury in England and Wales. BMF 1989; 296: 1305-1308.

3. Rozycki GS, Ochsner GM, Schmidt JA. et al. A prospective study of surgeon-performed ultra- sound as the primary adjuvant modality for injured patient assessment. F Trauma 1995; 39: 492-500.

4. Scalea TM, Rodriguez A, Chiu WC, Brenneman FD, Fallon WF Jr, Kato K, McKenney MG, Nerlich ML, Ochsner MG Jr, Yoshii H. Focused Assessment with Sonograpy for Trauma (FAST): Results from an International Consensus Conference. F Trauma 1999; 46(3): 444-472.

5. Dolich MO, McKenney MG, Varela JE, Compton RP, McKenney KL, Cohn S. 2576 ultrasounds for blunt abdominal trauma. F Trauma 2001; 50: 108112 .

6. Wherry DC, Potential of a hand-held ultrasound in assessment of the injured patient. Cardiovascular Surgery 1998; 6(6): 569-572.

7. American College of Surgeons, Advanced Trauma Life Support Student course Manual, 1997, Chicago.

8. McKenney MG, Martin L, Lentz K, Lopez C, Sleeman D, Aristide G, Kirton O, Nunez D, Najjar R, Namias N, Sosa J. 1000 consecutive ultrasounds for blunt abdominal trauma. FTrauma 1996; 40(4): 607-612.

9. Healey MA, Simons RK, Winchell RJ, Gosink BB, Casola G, Steele JT, Potenza BM, Hoyt DB. A prospective evaluation of abdominal ultrasound in blunt trauma: is it useful? $\mathcal{F}$ Trauma 1996; 40(6): 875-885.

10. Kahdi F, Udobi KF, Rodriguez, Chiu WC, Scalea TM. Role of ultrasonography in penetrating abdominal trauma: a prospective clinical study. $\mathcal{F}$ Trauma 2001; 50: 475-479.

11. Bain IH, Kirby RM, Cook AL, Oakley PA, Templeton J. Role of general surgeons in a British Trauma Centre. British Fournal of Surgery 1996; 83: $1248-1251$.

12. Thomas BT, Falcone RE, Vasquez D et al. Ultrasound evaluation of blunt abdominal trauma: program implementation, initial experience, and learning curve. F Trauma 1997; 42: 384-390. 